\title{
RLMS Algorithm for Fixed or Adaptive Beamforming
}

\author{
Jalal Abdulsayed Srar and Kah-Seng Chung \\ Department of Electrical and Computer Engineering, Curtin University of Technology, Perth, WA \\ jalal.srar@postgrad.curtin.edu.au, and k.chung@curtin.edu.au
}

\begin{abstract}
This paper presents a flexible method of achieving either fixed or self-adaptive antenna beamforming. It involves the use of the array image factor $A_{d}^{\prime}$, which interfaces the Recursive Least Squares (RLS) and Least Mean Squares (LMS) sections in cascade to form the RLMS beamforming algorithm. It is shown that an accurate fixed beam can be obtained by simply setting the elements of $A_{d}^{\prime}$ with prescribed values corresponding to the required direction. Moreover, the beam direction can also be made adaptive to automatically track the target signal. In this case, a simple method is described for estimating the element values of $A_{d}^{\prime}$ based on the estimated RLS output signal and tap weights. Computer simulation results verify these two modes of operation of the RLMS beamforming algorithm. Furthermore, the convergence performance of RLMS is shown to be quite insensitive to variations in SNR of the input signal as well as step sizes associated with the RLS and LMS sections.
\end{abstract}

Keywords-Fixed beam, RLMS algorithm, adaptive antenna array beam forming.

\section{INTRODUCTION}

Directional antennas are commonly used to exploit the spatial domain in an attempt to enhance coverage and capacity by minimizing interference in modern radio communications systems. Often, antennas with a prescribed beam pattern are used in fixed wireless links. More recently, adaptive or smart antennas are being adopted to automatically adjust and direct their beam patterns to the desired signals with nulls in the directions of interfering signals. The ability of these antennas to track their target signals quickly and accurately depends largely on the performance of the beamforming algorithm employed.

A seven-element electronically steerable parasitic array is described in [1]. It uses the simultaneous perturbation stochastic approximation algorithm to tune the reactance of each individual parasitic element so as to maximize the cross correlation coefficient between the desired and the received signals. In [2], dual orthogonal polarization diversity antenna elements are used in the front-end of discrete cylindrical lens array to generate a fan shaped fixed beam. On the other hand, different adaptive algorithms, including LMS and RLS, have been introduced for realizing steerable beam adaptive antennas [3]. Recently, variants of LMS and RLS have been investigated for improving the convergence speed and tracking ability in time varying environments. In [4], a variable step size LMS algorithm (VSSLMS) is applied for operation in the presence of nonstationary noise. A constrained constant modulus RLS algorithm is proposed for blind adaptive beamforming [5]. Also, a hybrid scheme which involves the use of sample matrix inversion to initialize the LMS algorithm to ensure fast convergence is presented in [6].

An adaptive configuration involving the use of RLS preceding LMS is presented in [7, 8]. This new RLMS set up is shown in Fig. 1. The array image factor $\boldsymbol{A}_{\boldsymbol{d}}^{\prime}$ is used to convert the RLS estimated output, $y_{R L S}$, back into individual array elements for further processing by the LMS section. Depending on how the element values of $\boldsymbol{A}_{\boldsymbol{d}}^{\prime}$ are derived, the same scheme can be used to provide either a fixed or self-adaptive beam pattern. This paper shows that an accurate fixed beam can be obtained by simply setting the elements of $\boldsymbol{A}_{\boldsymbol{d}}^{\prime}$ with prescribed values corresponding to the required direction. Also, any variations in operating condition and component tolerance could be compensated for by the adaptive adjustments of the RLS and LMS tap weights.

Increasingly, there are applications that require automatic tracking of moving target signals. In such situation, it is necessary for the antenna beam to self adjust itself so as to follow the angle of arrival (AoA) of the target signal. This can indeed be achieved using the RLMS scheme of Fig. 1. In this case, the element values of $\boldsymbol{A}_{\boldsymbol{d}}^{\prime}$ are made adaptable to varying AoA of the desired signal. In this paper, a simple method for estimating $A_{d}^{\prime}$ from the estimated RLS output signal and tap weights is presented. Furthermore, it is shown that the proposed scheme is effective for self-adaptive beam forming.

The rest of the paper is organized as follows. In section II, the use of prescribed element values for the array image factor $A_{d}^{\prime}$ for fixed beamforming is analyzed. Section III describes a simple method of deriving the element values of $\boldsymbol{A}_{\boldsymbol{d}}^{\prime}$ from the estimated RLS output signal $y_{R L S}$. Computer simulation results, obtained for an eight-element linear array, are presented in Section IV for both fixed and self-adaptive beam forming. Finally, Section V concludes the paper.

\section{FIXED BEAMFORMING USING THE RLMS ALGORITHM}

Fig. 1 shows the block diagram of an $N$-isotropic element adaptive linear array, which employs RLMS as its beam forming algorithm.

Let the desired signal $s_{d}(t)$ and a cochannel interference $s_{i}(t)$, 


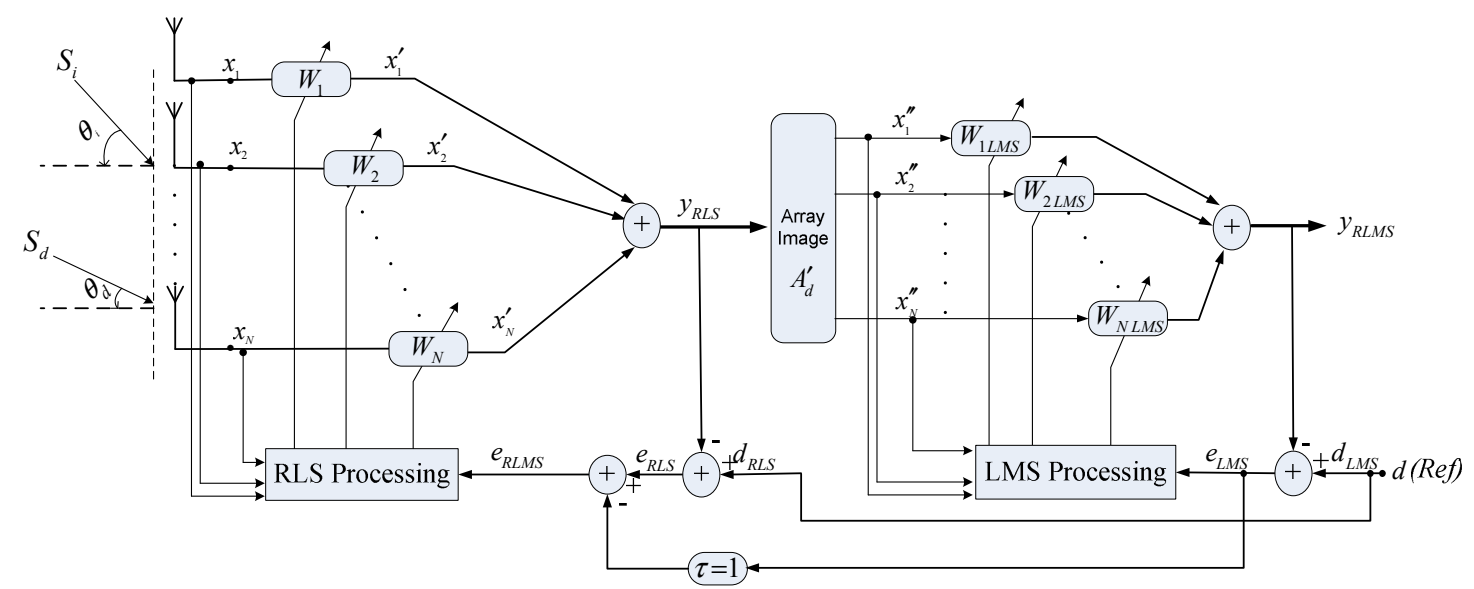

Figure 1. A configuration of the RLMS algorithm

both originated from a distance, are impinging on the array at an angle $\theta_{d}$ and $\theta_{i}$, respectively, as shown in Fig. 1. The resulting outputs of the individual antenna elements in the presence of Additive White Gaussian Noise (AWGN), $\mathbf{n}(t)$ of variance $\sigma^{2}$ can be expressed as

$$
\begin{aligned}
\mathbf{x}(t) & =\left[x_{1}(t), x_{2}(t), \ldots, x_{N}(t)\right]^{H} \\
& =\mathbf{A}_{d} s_{d}(t)+\mathbf{A}_{i} s_{i}(t)+\mathbf{n}(t)
\end{aligned}
$$

where $\boldsymbol{A}_{d}$ and $\boldsymbol{A}_{i}$ are the array factors for the desired signal and the cochannel interference, respectively and $(\bullet)^{H}$ denotes the complex conjugate transpose.

By referencing with respect to the first element, $\boldsymbol{A}_{d}$ and $\boldsymbol{A}_{i}$ are given by

$$
\begin{aligned}
& \boldsymbol{A}_{d}=\left[1, e^{-j \psi_{d}}, e^{-2 j \psi_{d}}, \ldots, e^{-(N-1) j \psi_{d}}\right]^{H} \\
& \boldsymbol{A}_{i}=\left[1, e^{-j \psi_{I}}, e^{-2 j \psi_{I}}, \ldots, e^{-(N-1) j \psi_{I}}\right]^{H}
\end{aligned}
$$

with $\psi_{d}=2 \pi\left(\frac{d \sin \left(\theta_{d}\right)}{\lambda}\right)$ and $\psi_{i}=2 \pi\left(\frac{d \sin \left(\theta_{i}\right)}{\lambda}\right)$, where $d$ is the antenna element spacing, and $\lambda$ is the carrier wavelength.

According to Fig. 1, the input stage of the RLMS scheme is based on the RLS algorithm with its weight vector at the $(j+1)^{\text {th }}$ iteration updated according to [9]

$$
\boldsymbol{W}_{R L S}(j+1)=\boldsymbol{W}_{R L S}(j)+\boldsymbol{p}(j+1) \boldsymbol{X}(j) e_{R L S}^{*}(j)
$$

where $\boldsymbol{p}(j+1)$ is an arbitrary symmetric positive definite matrix given by

$$
\boldsymbol{p}(j+1)=\frac{1}{\alpha}\left[\boldsymbol{p}(j)-\frac{\boldsymbol{p}(j) \boldsymbol{X}(j) \boldsymbol{X}^{H}(j) \boldsymbol{p}(j)}{\alpha+\boldsymbol{X}^{H}(j) \boldsymbol{p}(j) \boldsymbol{X}(j)}\right]
$$

$\boldsymbol{p}(j)$ is initialized as $\delta^{-1} \boldsymbol{I}$, with $\delta$ being a small positive constant, $\alpha$ is the forgetting factor and $\boldsymbol{I}$ is an $N \times N$ identity matrix. In this paper, $\alpha$ is assumed to be equal to 1 .

Now, the RLS output at the $j^{\text {th }}$ iteration can be expressed as

$$
y_{R L S}(j)=\boldsymbol{W}_{R L S}^{H}(j) \boldsymbol{X}(j)
$$

where $\boldsymbol{W}$ denotes the the weight vector $\boldsymbol{W}(\cdot)$.

From this estimated RLS output signal, the input signal vector for the LMS section can be obtained, such that

$$
\boldsymbol{X}_{L M S}=\boldsymbol{A}_{\boldsymbol{d}}^{\prime} y_{R L S}
$$

The LMS weight vector is updated according to

$$
\boldsymbol{W}_{L M S}(j+1)=\boldsymbol{W}_{L M S}(j)+\mu \boldsymbol{X}_{L M S}(j) e_{L M S}(j) \quad 0<\mu<\mu_{0}
$$

where $\mu_{0}$ is a positive constant with its value dependent on the input signal statistics.

Finally, the output of the RLMS beamformer is given by

$$
\begin{aligned}
y_{R L M S} & =\boldsymbol{W}_{L M S}^{H} \boldsymbol{X}_{\text {LMS }} \\
& =\boldsymbol{W}_{L M S}^{H} y_{R L S} \boldsymbol{A}_{\boldsymbol{d}}^{\prime}
\end{aligned}
$$

Equation (9) indicates the central role played by the image array factor $\boldsymbol{A}_{\boldsymbol{d}}^{\prime}$ in beamforming using the RLMS algorithm. Now, by prescribing the individual elements of $\boldsymbol{A}_{\boldsymbol{d}}^{\prime}$ with values corresponding to the required AoA, the resulting output will contain only those signal components "selected" by $\boldsymbol{A}_{\boldsymbol{d}}^{\prime}$. For example, it is only required to set $\boldsymbol{A}_{d}^{\prime}=\boldsymbol{A}_{d}$ in order to obtain a fixed beam pointing in the direction of $\theta_{d}$.

\section{ESTIMATION OF ARRAY IMAGE FACTOR $\boldsymbol{A}_{\boldsymbol{d}}^{\prime}$}

For self-adaptive beamforming, it requires that $\boldsymbol{A}_{\boldsymbol{d}}^{\prime}$ be adjusted automatically to always tracking the AoA of the desired signal. A simple method for estimating $\boldsymbol{A}_{\boldsymbol{d}}^{\prime}$ is now described. 
Consider the array inputs to the RLS section by rearranging (1) to become

$$
\begin{gathered}
x_{1}(t)=A_{d 1} s_{d}(t)+A_{i 1} s_{i}(t)+n(t) \\
x_{2}(t)=A_{d 2} s_{d}(t)+A_{i 2} s_{i}(t)+n(t) \\
\vdots \quad \vdots \quad \vdots \quad \vdots \quad \vdots \\
x_{N}(t)=A_{d N} s_{d}(t)+A_{i N} s_{i}(t)+n(t)
\end{gathered}
$$

where $A_{d 1}, A_{d 2}, A_{d 3}, \ldots \ldots, A_{d N}$ are the array factor elements. by

The output of the individual RLS tap weights are then given

$$
\begin{aligned}
& x_{1}^{\prime}(t)=w_{1}\left[A_{d 1} s_{d}(t)+A_{i 1} s_{i}(t)+n(t)\right] \\
& x_{2}^{\prime}(t)=w_{2}\left[A_{d 2} s_{d}(t)+A_{i 2} s_{i}(t)+n(t)\right] \\
& \vdots \quad \vdots \quad \vdots \quad \vdots \\
& x_{N}^{\prime}(t)=w_{N}\left[A_{d N} s_{d}(t)+A_{i N} s_{i}(t)+n(t)\right]
\end{aligned}
$$

When the RLS stage converges, its output $y_{R L S}$, approaches the desired signal $s_{d}(t)$. At the same time, both the interference $s_{i}(t)$ and noise in (11) are being suppressed. As a result, the individual elements of $\boldsymbol{A}_{\boldsymbol{d}}^{\prime}$ are approximated by

$$
\begin{gathered}
A_{d 1}^{\prime} \simeq \frac{x_{1}^{\prime}(t)}{w_{1}(t) y_{R L S}(t)} \\
A_{d 2}^{\prime} \simeq \frac{x_{2}^{\prime}(t)}{w_{2}(t) y_{R L S}(t)} \\
\vdots \quad \vdots \quad \vdots \\
A_{d N}^{\prime} \simeq \frac{x_{N}^{\prime}(t)}{w_{N}(t) y_{R L S}(t)}
\end{gathered}
$$

The error convergence of the RLMS algorithm using the estimated $\boldsymbol{A}_{\boldsymbol{d}}^{\prime}$ is analyzed in [8].

\section{Simulation Results}

The ability of the RLMS algorithm to realize either fixed or self-adaptive beamforming has been investigated by means of MATLAB simulations. For these simulations, the following parameters are common to both types of beamforming:

- A linear array consisting of 8 isotropic antenna elements spaced half a carrier wavelength apart.

- A Binary Phase Shift Keying (BPSK) signal arriving at a specified angle of arrival.

- The channel is AWGN of zero mean and variance $\sigma^{2}$.

- All the weight vectors are initially set to zero.

- Each simulation run involves 100 iterations.

\section{A. Fixed beamforming}

For fixed beamforming, individual elements of $\boldsymbol{A}_{\boldsymbol{d}}^{\prime}$ are being assigned values pre-calculated using (2) according to the required direction. Fig. 2 shows the fixed beam patterns obtained when the direction is set to either $-20^{\circ}, 0^{\circ}, 20^{\circ}, 40^{\circ}$ or $60^{\circ}$. In this case, the input signal with an $S N R=10 \mathrm{~dB}$ is arriving at angle $\theta_{d}$ corresponds to the respective specified direction, i.e., $-20^{\circ}, 0^{\circ}, 20^{\circ}, 40^{\circ}$ or $60^{\circ}$. Except for the case of $\theta_{d}=60^{\circ}$, the beam patterns are almost identical with a worst case side lobe gain of $-13 \mathrm{~dB}$.

Next, the beam resolution that could be achieved with the proposed scheme is investigated. The resulting beam patterns achieved for direction set at either $-2^{\circ}, 0^{\circ}, 1^{\circ}$ or $5^{\circ}$ are shown in Fig. 3. These results indicate that it is possible to differentiate very small difference in beam direction. Also, the proposed scheme is shown to be robust in the presence of interfering signals occurring outside the beam width. For example, the beam pattern obtained for the specified direction of $30^{\circ}$, in the presence of three interfering signals of equal amplitude as the desired signal and arriving at $\theta_{i}=-50^{\circ},-30^{\circ}$ and $45^{\circ}$, is shown in Fig. 4. This result demonstrates that the adaptive nature of the RLMS algorithm helps to create null responses at the angles of occurrence of the interfering signals.

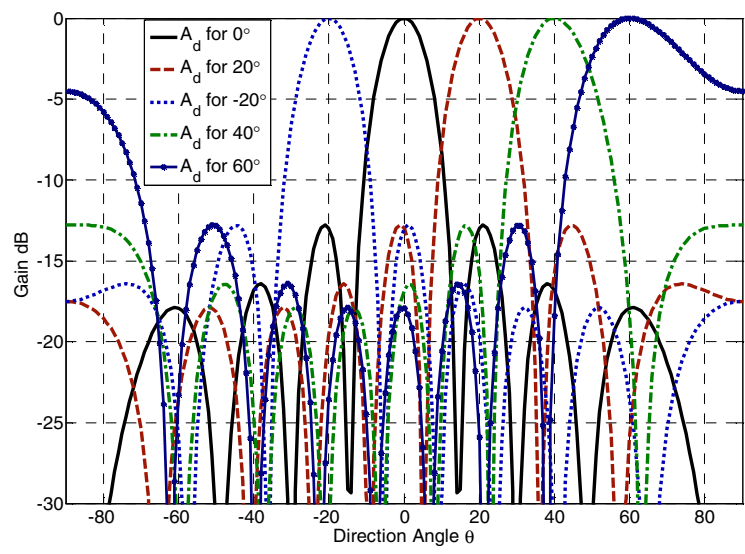

Figure 2. The beam patterns obtained at five large angles of $\theta_{d}$ $\left(-20^{\circ}, 0^{\circ}, 20^{\circ}, 40^{\circ}\right.$ and $\left.60^{\circ}\right)$

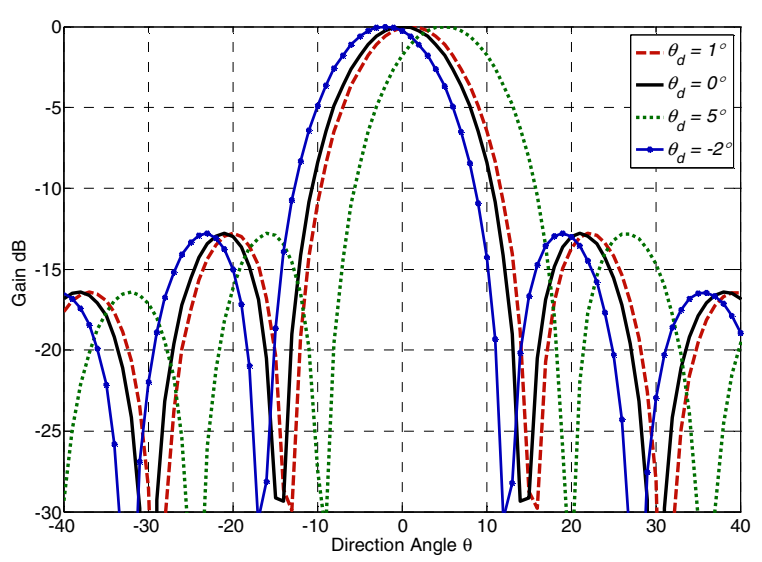

Figure 3. Beam patterns obtained at four small angles of $\theta_{d}$

$$
\left(-2^{\circ}, 0^{\circ}, 1^{\circ} \text { and } 5^{\circ}\right)
$$


Furthermore, consider the beam direction is fixed at $0^{\circ}$ and the input signal has an SNR of $10 \mathrm{~dB}$. The output signal to interference plus noise ratio, $\operatorname{SINR}_{o}$, achieved by this signal as a function of iterations, as measured at five different values of AoA, i.e., $0^{\circ}, 10^{\circ}, 20^{\circ},-10^{\circ}$ or $-20^{\circ}$, is shown in Fig. 5. Fig. 5 shows the rapid convergence of $\operatorname{SINR}_{o}$ to its corresponding steady value within ten iterations. As to be expected, the maximum $\operatorname{SINR}_{o}$ of $18 \mathrm{~dB}$ is achieved when the signal arrives at the beam direction of $0^{\circ}$. As the AoA of the signal deviates from the beam direction, the achievable $\operatorname{SINR}_{o}$ is also reduced in accordance with that indicated by the beam pattern.

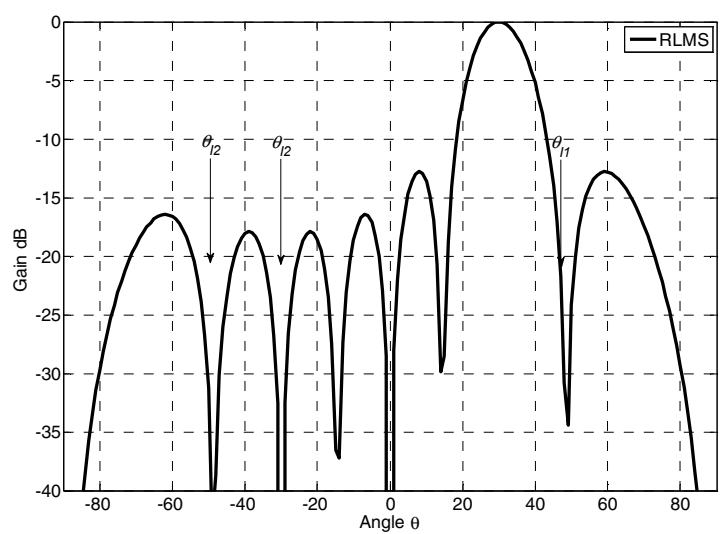

Figure 4. The beam patterns obtained in the presence of three equalamplitude interfering signals at $\theta_{I 1}=45^{\circ}, \theta_{I 2}=-30^{\circ}$ and $\theta_{I 3}=-50^{\circ}$

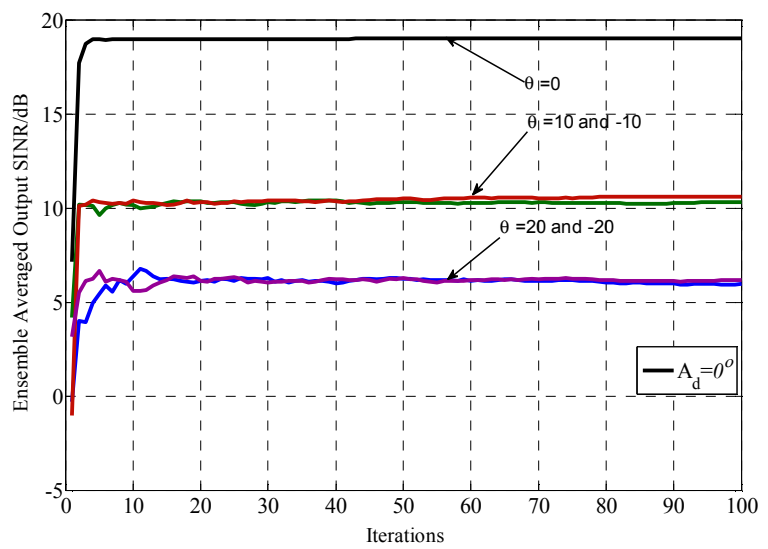

Figure 5. Output SINR versus iterations for an input signal of $S N R=10 \mathrm{~dB}$ as measured at five different values of AoA. The input signal arrives at $0^{\circ}$ and the RLS step size used is $\delta=0.05$

\section{B. Self-adaptive beam forming}

In this section, the performance of the RLMS algorithm for self-adaptive beamforming based on $\boldsymbol{A}_{\boldsymbol{d}}^{\prime}$ estimated from (12) is investigated under different operating conditions.

\section{1) Error convergence}

The convergence of the RLMS algorithm achieved with the use of an external reference signal has been studied based on the ensemble average square error $\left(\tilde{e}^{2}\right)$ obtained from 100 individual simulation runs. The effects of input $S N R$ on the convergence of the RLMS algorithm $(\delta=0.05, \mu=0.075)$ are shown in Fig. 6 for three different values of $S N R$, i.e., $5 d B, 10 d B$, and $15 d B$. The convergence of the RLS algorithm ( $\delta=0.05$ ) operating on its own is also included here for comparison. It is observed that both algorithms achieved similar convergence speed but RLS suffers from a higher error floor particularly at lower $S N R$. For $S N R \geq 10 \mathrm{~dB}$, RLMS converges slightly faster than RLS, at less than 10 iterations. Moreover, RLMS converges to almost the same error floor for all the three $S N R$ values considered.

Furthermore, simulations have also been carried out using different combinations of $\delta(0.05,0.5$, and 1$)$ and $\mu(0.001$, 0.01 and 0.1 ). The results obtained (not shown here) verify that the operation of RLMS is not very sensitive to the choice of these step sizes.

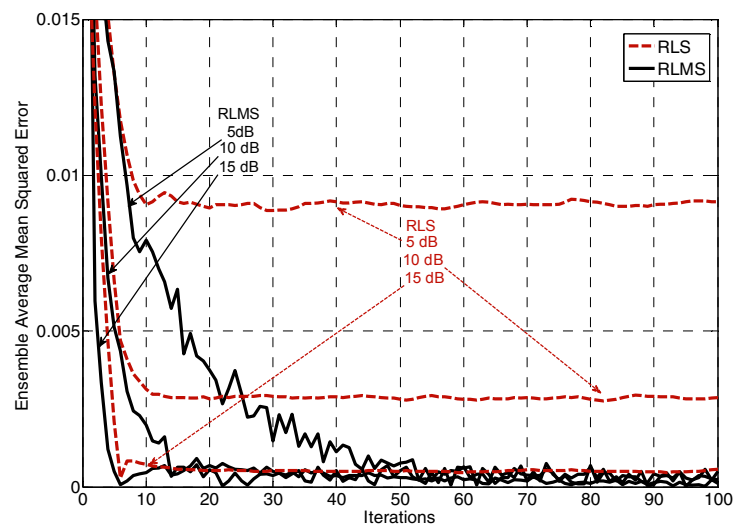

Figure 6. The convergence of RLS and RLMS with $\delta=0.05$ and $\mu=0.075$ for three different values of input $S N R$

\section{2) Performance in the presence of multi-interference} signals

Consider the desired signal arriving from the direction $\theta_{d}=0$ is corrupted by four cochannel interfering signals of four equal-amplitude with AoA of $20^{\circ}, 45^{\circ},-30^{\circ}$ and $-50^{\circ}$.

From the beam pattern of Fig. 7, it is observed that the RLMS algorithm is effective in suppressing close-in interference, say $\theta_{i}=20^{\circ}$, with a rejection ratio of $22 \mathrm{~dB}$. Furthermore, the other three interfering signals with larger AoAs are being suppressed by a greater degree. 


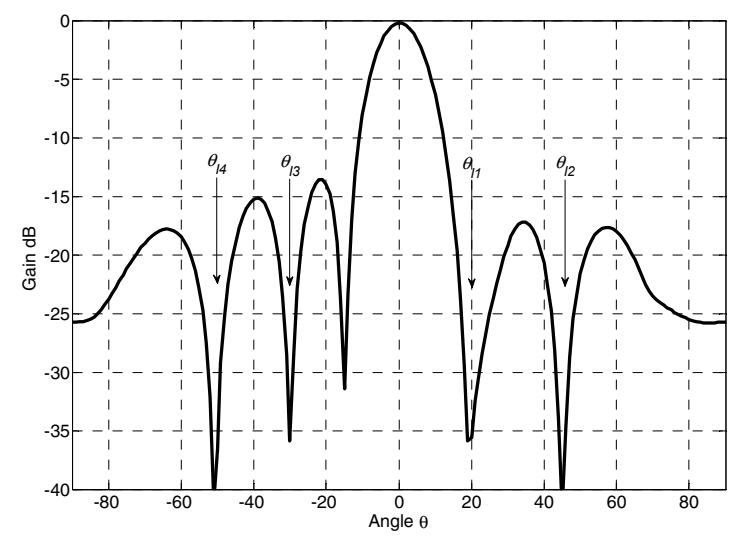

Figure 7. The beam patterns obtained in the presence of four equalamplitude interfering signals with AoA at

$$
\theta_{I 1}=20^{\circ}, \theta_{I 2}=45^{\circ}, \theta_{I 3}=-30^{\circ} \text { and } \theta_{I 4}=-50^{\circ}
$$

\section{3) Tracking performance}

The ability of the RLMS algorithm in tracking sudden interruptions in the input signal is investigated by examining the behavior of its error signal $e_{L M S}$. For this study, the input signal is interrupted periodically, i.e., 25 out of every 100 iterations. The resulting tracking performance of RLMS is shown in Fig. 8, which indicates that the mean square error $\xi$ increases very rapidly each time the input is either switched on or off. This verifies the fast response of RLMS to sudden changes in input signal. Unlike the response for RLS, which is also shown in Fig. 8 for comparison purposes, the mean square error $\xi$ associated with RLMS remains low despite the interruption in the input signal.

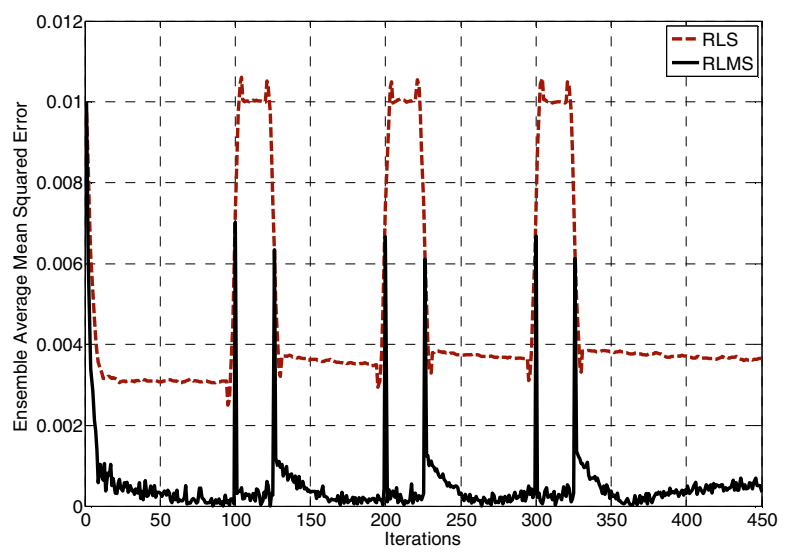

Figure 8. Tracking performance comparison of RLS and RLMS with $\delta=0.05, \mu=0.075$ and input $S N R=10 d B$

\section{CONCLUSIONS}

A new array beamforming algorithm, called RLMS, has been investigated. The study highlights the central role played by the array image factor $\boldsymbol{A}_{d}^{\prime}$, which is responsible for reproducing the various array component signals from the intermediate RLS output for further processing by the LMS algorithm. Computer simulation results have verified that either fixed or self-adaptive beam forming could be realized depending on the actual method used to obtain the values of $\boldsymbol{A}_{d}^{\prime}$.

To obtain fixed beamforming, it is only necessary to assign $A_{d}^{\prime}$ with prescribed values calculated for the required direction. Simulation results have shown that it is possible to differentiate very small difference in beam direction. Furthermore, the beam pattern can be directed to cover over a wide and precise range.

Furthermore, a simple method for estimating the element values of $\boldsymbol{A}_{d}^{\prime}$ has also been presented. Through its use, it is possible for the RLMS algorithm to realize self-adaptive beamforming. Computer simulations have verified that the interference suppression capability and convergence performance of the proposed scheme are quite insensitive to changes in input SNR as well the step sizes adopted for the RLS and LMS sections.

\section{REFERENCES}

[1] A. Osseiran and A. Logothetis, "A Method for Designing Fixed Multibeam Antenna Arrays in WCDMA Systems", Antennas and Wireless Propagation Letters, vol. 5, pp. 41-44 Dec. 2006.

[2] D. Popovic and Z. Popovic, "Multibeam antennas with polarization and angle diversity", Antennas and Propagation, IEEE Transactions on, vol. 50, pp. 651-657, 2002.

[3] F.-B. Ueng, J.-D. Chen, and S.-H. Cheng, "Smart Antenna for Multiuser DS/CDMA Communication in Multipath Fading Channels", IEICE Transaction Communication, vol. E88, pp. 2944-2954, Jul 2005.

[4] A. Gupta and S. Joshi, "Variable Step-Size LMS Algorithm for Fractal Signals", Signal Processing, IEEE Transactions on, vol. 56, pp. 14111420, 2008

[5] W. Lei and R. C. de Lamare, "Constrained Constant Modulus RLS-based Blind Adaptive Beamforming Algorithm for Smart Antennas," 4th International Symposium on Wireless Communication Systems, pp. 657661, Trondheim, Norway, 2007.

[6] R. M. Shubair, A. Merri, and W. Jessmi, "Improved adaptive beamforming using a hybrid LMS/SMI approach", Second IFIP International Conference on Wireless and Optical Communications Networks, pp. 603-606, Dubai, United Arab Emirates, 2005.

[7] J. A. Srar and K.-S. Chung, "Performance of RLMS Algorithm in Adaptive Array Beam Forming", 11th IEEE International Conference on Communication Systems Guangzhou, China, Nov. 2008.

[8] J. A. Srar and K.-S. Chung, "Adaptive Array Beam Forming Using a Combined RLS-LMS Algorithm", The 14th Asia-Pacific Conference on Communications, Tokyo, Japan, Oct. 2008.

[9] L. C. Godara, "Application of antenna arrays to mobile communications. II. Beam-forming and direction-of-arrival considerations," Proceedings of the IEEE, vol. 85, pp. 1195-1245, 1997. 